Z Gerontol Geriat 2016 · 49:765

DOI 10.1007/s00391-016-1139-5

Online publiziert: 19. Oktober 2016

๑) Springer-Verlag Berlin Heidelberg 2016

CrossMark

\section{Originalpublikation}

Køber L, Thune JJ, Nielsen JC et al (2016) Defibrillator implantation in patients with nonischemic systolic heart failure. $\mathrm{N}$ Engl J Med. doi:10.1056/NEJMoa1608029

Einleitung. In den Leitlinien ist die prophylaktische Implantation von Defibrillatoren („implantable cardioverter-defibrillator", ICD) bei Herzinsuffizienz und eingeschränkter Linksventrikelfunktion etabliert, obwohl die Evidenz für Patienten mit zugrunde liegender koronarer Herzkrankheit (KHK) höher ist als für Patienten mit einer Herzinsuffizienz anderer Genese. Diese randomisierte Studie wurde daher an Patienten mit chronischer Herzinsuffizienz und reduzierter Linksventrikelfunktion anderer Genese als einer KHK mit oder ohne ICD-Implantation durchgeführt.

Methode. Ausgeschlossen wurden Patienten unter einer Dialyse oder mit tachykardem Vorhofflimmern in Ruhe. Entsprechend den Ein- und Ausschlusskriterien passende Patienten, die in die Teilnahme an der Studie einwilligten, wurden, 1:1 randomisiert, der Standardtherapie oder der zusätzlichen Behandlung mit einem ICD zugeführt und 2 Jahre lang nachuntersucht.

Resultate. Zwischen Februar 2008 und Juni 2014 wurden 556 Patienten der ICDGruppe und 560 der Standardtherapiegruppe zugeordnet. Das Durchschnittsalter der Patienten betrug 64 Jahre (Range 56 bis 72 Jahre) in der ICD- und 63 Jahre (Range 56 bis 70 Jahre) in der Standardtherapiegruppe; es waren $27 \%$ resp. $28 \%$ der Teilnehmenden weiblich. In der durchschnittlich 67,6 Monate dauern-

Peter Dovjak

Akutgeriatrie, Salzkammergut-Klinikum, Gmunden, Österreich

\title{
Implantierte Defibrillatoren selektiv anwenden
}

den Nachuntersuchungsperiode starben 21,6\% Patienten der ICD- und 23,4\% der Standardtherapiegruppe. Sowohl bezüglich der Gesamtmortalität, kardiovaskulärer Mortalität und auch der Todesfälle durch plötzlichen Herztod fanden sich keine signifikanten Unterschiede. In der Subgruppenanalyse waren die Ergebnisse für Ältere über 68 Jahre signifikant schlechter als für Jüngere.

Diskussion. Die Behandlung mit einem ICD bei herzinsuffizienten Patienten ohne KHK bringt keinen signifikanten Überlebensvorteil. Für Ältere weist der Unterschied zur Standardbehandlung überdies einen Nachteil auf. Die Infektionsrate in der ICD-Gruppe ist beachtlich.

\section{Kommentar}

Wie McMurray [1] im Editorial der gleichen Ausgabe des New England Journal of Medicine betont, beruhten die Empfehlungen in den Leitlinien lediglich auf den Ergebnissen von Metaanalysen. Auch dürfte die konsequente Verwendung von ausreichend dosierten Medikamenten zur Behandlung der Herzinsuffizienz wie $\beta$-Rezeptoren-Blocker, Angiotensinkonversionsenzym(ACE)-Hemmer, Angiotensin(AT)-1-Antagonisten und Mineralokortikoidrezeptorantagonisten sich zugunsten der Standardtherapiegruppe in der präsentierten Studie ausgewirkt haben. Jedenfalls sollten Patienten, die offensichtlich nicht profitieren, keiner ICD-Implantation zugeführt werden. Bei Älteren sollte die Indikationsstellung besonders kritisch erfolgen.

\section{Korrespondenzadresse}

Prim. Dr. P. Dovjak

Akutgeriatrie, Salzkammergut-Klinikum Miller-von-Aichholz-Str. 49, 4810 Gmunden, Österreich

Peter.dovjak@gespag.at

Interessenkonflikt. P. Dovjak gibt an, dass kein Interessenkonflikt besteht.

\section{Literatur}

1. McMurray JJ (2016) The ICD in heart failure time for a rethink? N Engl J Med. doi:10.1056/ NEJMe1609826 